\title{
Isolated Adenoma with Neuroendocrine Differentiation Involving Both the Facial Nerve and Parotid Gland
}

\author{
Ali K. Mahrous ${ }^{1}$, James R. Tysome' ${ }^{1}$, Alison Marker², David A. Moffat ${ }^{1}$ \\ ${ }^{1}$ Department of ENT-Skull Base Surgery, Addenbrookes NHS Foundation Trust, Cambridge University \\ Hospitals, Cambridge, UK \\ ${ }^{2}$ Department of Pathology, Addenbrookes NHS Foundation Trust, Cambridge University Hospitals, \\ Cambridge, UK \\ Email: alikhalaf@doctors.org.uk
}

Received 12 February 2014; revised 4 March 2014; accepted 24 March 2014

Copyright (C) 2014 by authors and Scientific Research Publishing Inc.

This work is licensed under the Creative Commons Attribution International License (CC BY). http://creativecommons.org/licenses/by/4.0/

(c) (i) Open Access

\begin{abstract}
Objective: To report an extremely rare case of neuroendocrine tumour with simultaneous involvement of both the facial nerve and the deep lobe of parotid gland. Method: case report and English language literature review concerning neuroendocrine neoplasm involving the facial nerve with emphasis on clinical presentation. Results: We report a unique case of adenoma with neuroendocrine differentiation which involved both the mastoid segment of the right facial nerve and also the deep lobe of the parotid gland on the ipsilateral side. Both tumours were not contiguous and were anatomically separate from each other. A CT scan of the whole body revealed no other neuroendocrine tumours. Conclusion: To the best of our knowledge, this is the first report in the English language literature of a neuroendocrine tumour to involve both the vertical mastoid segment of the facial nerve with simultaneous involvement of the deep lobe of parotid gland as a separate lesion.
\end{abstract}

\section{Keywords}

Neuroendocrine; Adenoma; Middle Ear; Facial Nerve; Parotid

\section{Introduction}

Middle ear glandular neoplasms are seldom the source of a middle ear mass. Hyams [1] was the first to describe

"Corresponding author.

How to cite this paper: Mahrous, A.K., et al. (2014) Isolated Adenoma with Neuroendocrine Differentiation Involving Both the Facial Nerve and Parotid Gland. International Journal of Otolaryngology and Head \& Neck Surgery, 3, 101-105. 
a series of these tumours in 1976. He designated these tumours as middle ear adenomas (MEA). In 1980, Murphy et al. [2] described a similar, if not identical, tumour and designated it a carcinoid tumour because of the ultrastructural evidence of neuroendocrine differentiation.

This is a unique clinical scenario and to our knowledge, it has not been reported in the literature before.

\section{Case Report}

A 66-year-old Caucasian lady presented with fluctuating right-sided lower motor facial palsy and intermittent discomfort around her right ear for couple of years. It was initially thought to be a Bell's palsy by the referring clinician as it did respond to high dose steroids. Nevertheless, the recovery of the facial palsy was only partial to House-Brackmann (HB) grade III. It eventually progressed to complete paralysis (HB VI). Interestingly, her pain had become severe enough to warrant hospital admission. Clinical examination revealed normal tympanic membranes on both sides and a HB grade VI facial palsy on the right side. The rest of the neuro-otological examination was unremarkable. A pure tone audiogram showed symmetrical high-frequency SNHL. High resolution CT scan of the temporal bone revealed widening of the right mastoid vertical segment of the facial nerve canal with soft-tissue mass (Figure 1).

The case was discussed in our Multidisciplinary Skull Base Team meeting and, based on both the clinical and radiological findings; it was suspected to be a facial schwannoma of the vertical segment of the nerve. It is to be noted that initially there was no radiological evidence of a deep lobe parotid tumour. Management options were discussed and in view of the presence of a complete facial palsy, it was decided to recommend surgical excision of the tumour. At surgery the appearance of the tumour and its consistency was not typical of a facial schwannoma and it was possible to find a plane of cleavage and to carefully dissect the tumour from the nerve. The distal facial nerve at the stylomastoid foramen was normal. A frozen section histopathological examination of the specimen was inconclusive. The formal histopathological examination was reported as adenoma with neuroendocrine differentiation and was as follows: "the histology showed features consistent with a neuroendocrine tumour. There appeared to be some luminal secretion in the small glands in places and mitoses were sparse and there were no features of overt malignancy. The neoplasm is consistent with a benign adenoma Figures 2 and 3. It may have arisen from the middle ear sinus tympani or mastoid air cells in the vicinity of the facial canal. In the histological differential diagnosis is a low grade neuroendocrine carcinoma but the long clinical history is against this and the other diagnosis could be ectopic pituitary adenoma but the immuno-profile is not entirely consistent”.

The post-operative course was uneventful and facial function improved to HB grade IV. This was only maintained for 3 months, however, when her facial function deteriorated again to grade HB VI, a repeat MRI scan Figure 4 revealed a tumour in the deep lobe of right parotid gland $13 \mathrm{~mm}$ in diameter and radiologically separate from the original tumour.

The case was discussed again in the MDT skull base meeting where a decision was taken to surgically remove the tumour in the deep lobe of parotid and remove the vertical mastoid segment of the facial nerve followed by cable grafting with sural nerve and a "baby-sitting" facio-hypoglossal anastomosis technique. The histopatho-

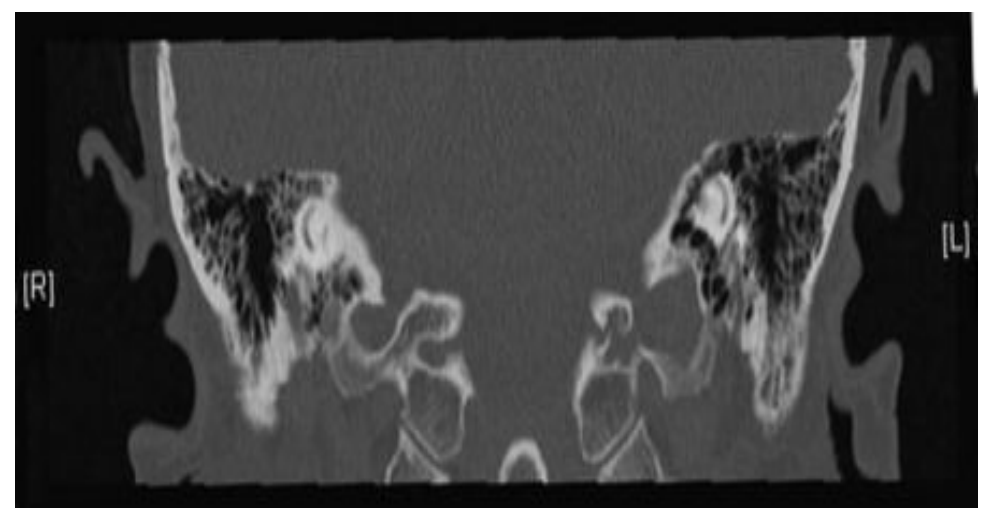

Figure 1. CT temporal bone shows widening of the mastoid segment of facial nerve canal—right side. 


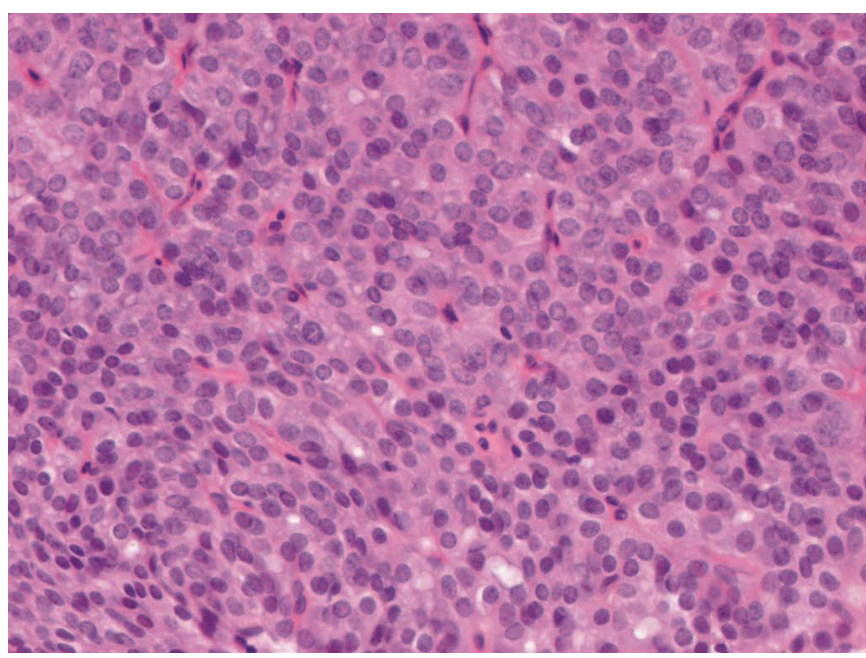

Figure 2. Histopathological features of the neuroendocrine tumour (H\&E stain).

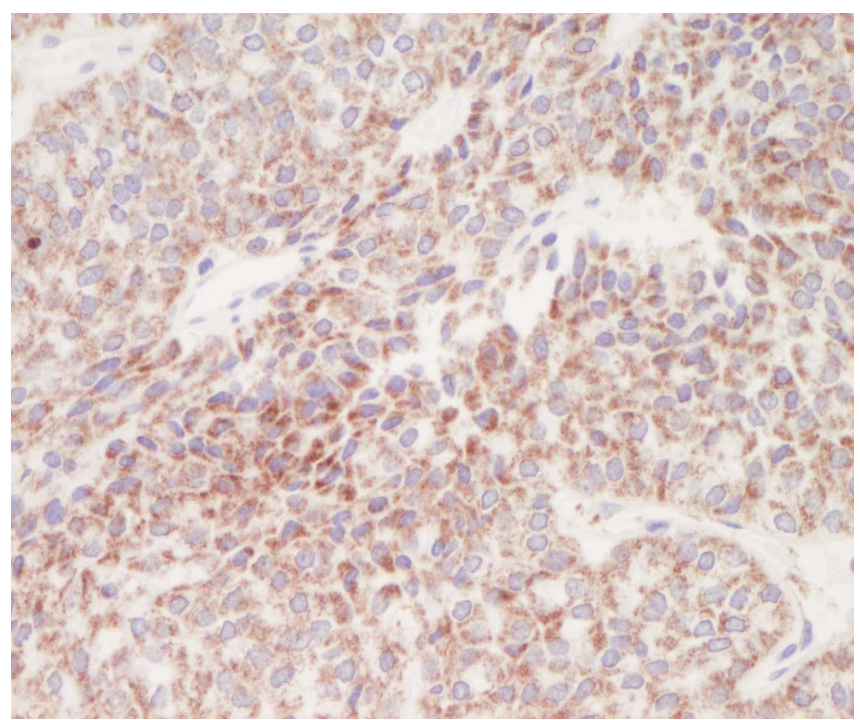

Figure 3. Immunostaining for chromogranin A. Tumour cells showed weak to moderate granular cytoplasmic positivity.

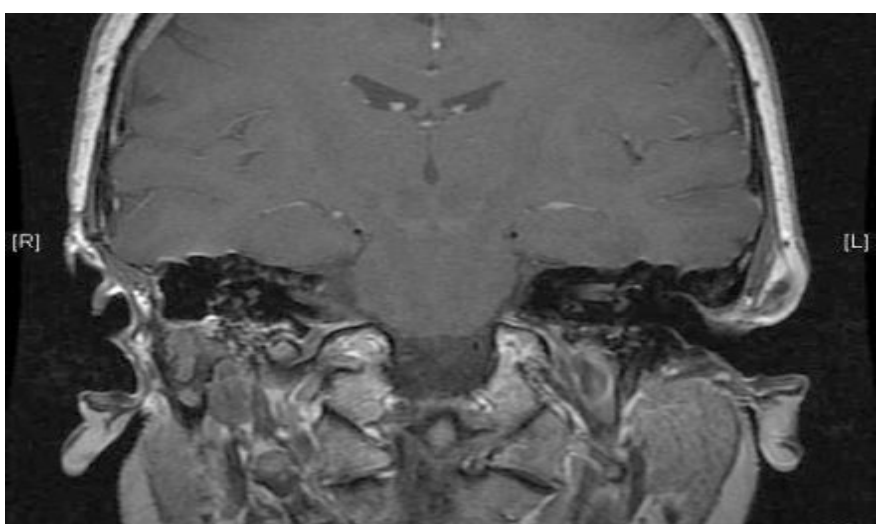

Figure 4. MRI shows a tumour in the right deep lobe of the parotid gland. 
logical features of the parotid tumour were exactly the same as the original facial nerve tumour. It was thought that these tumours might be associated with other neuroendocrine tumours as part of the multiple neuroendocrine tumour syndrome. Therefore, a post-operative whole body CT scan was performed which revealed no evidence of other neuroendocrine pathology. The patient is still under close review in the outpatient clinic.

\section{Discussion}

This appeared to be a unique case which to our knowledge has not been reported in the literature before.

The histopathologist suggested that the tumour in close proximity to the descending facial nerve could have arisen from the mucosal lining of the sinus tympani or retrofacial air cell system.

Middle ear adenomas are rare but are in the differential diagnosis of middle ear tumours. Hyams [1] was the first to describe a series of these tumours in 1976.

In 1980, Murphy et al. [2] described a similar tumour and called it a carcinoid tumour because of the ultrastructural evidence of a neuroendocrine differentiation.

Middle ear glandular neoplasms can present with both a neuroendocrine and epithelial differentiation. This has led some early authors to maintain that neuroendocrine (carcinoid) tumours and middle ear adenomas were different tumours [3]. However, most authorities currently think that there is a single primary low-grade glandular neoplasm of the middle ear. This has led most authorities to conclude that a middle ear adenoma, or the variant neuroendocrine adenoma, was a preferred nomenclature as opposed to that of a carcinoid tumour since these terms imply a benign behaviour, which is consistent with the vast majority of cases [4].

Carcinoid tumours of the lung are thought to originate from enterochromaffin cells (Kulchitsky cells), which are neuroendocrine normal cells present in the lung. However, epithelial cells with neuroendocrine characteristics are not noted in the middle ear cavity. An undifferentiated pluripotential endodermal stem cell may still be present within the surface mucosa, giving rise to carcinoid neoplasms similar to the Kulchitsky cell [5]. Hyams and Michaels were the first to hypothesize that middle ear adenomas originated from the mucosal epithelium of the middle ear, but the lack of evidence for surface epithelial derivation leads to the consideration of a stromal precursor [1]; the stroma of the middle ear derives from the mesoderm and the neural crest. The neural crest gives rise to parts of the ossicular chain and the three primary paraganglia. Positive immunohistochemical staining for neuroendocrine tissue, neuron-specific enolase, chromogranin, and/or synaptophysin suggest that adenoma of the temporal bone originates from neuroectoderm.

Epithelial and exocrine characteristics are not normal features of these cells and it is plausible that a neuroendocrine neoplasm of the middle ear may originate from a neural crest-derived stem cell [4].

In our case, whilst macroscopically it appeared that the two lesions were separate it is not clear whether the tumour around the vertical mastoid segment of the right facial nerve was completely separate from the parotid tumour and their presence was a pure coincidence or whether it was a skip lesion of the same pathology. The histopathological similarities of the two tumours raise the suspicion that it was the latter.

\section{Summary}

- Neuroendocrine tumour is a rare neoplasm to involve the facial nerve.

- Our case is unique as not only has it involved the mastoid segment of the facial nerve but also it did involve the deep lobe of the ipsilateral parotid gland as a skip lesion with no anatomical continuity.

- Recurrent facial nerve palsy should raise our suspicion and lower our threshold of scanning the temporal bone.

\section{References}

[1] Hyams, V.J. and Michaels, L. (1976) Benign Adenomatous Neoplasm (Adenoma) of the Middle Ear. Clinical Otolaryngology and Allied Sciences, 1, 17-26. http://dx.doi.org/10.1111/j.1365-2273.1976.tb00637.x

[2] Murphy, G.F., Pilch, B.Z., Dickersin, G.R., Goodman, M.L. and Nadol, Jr., J.B. (1980) Carcinoid Tumor of the Middle Ear. American Journal of Clinical Pathology, 73, 816-823.

[3] Friedmann, I. (1998) Middle Ear Adenoma. Histopathology, 32, 279-280. http://dx.doi.org/10.1046/j.1365-2559.1998.0372b.x

[4] Saliba, I. and Evrard, A.-S. (2009) Middle Ear Glandular Neoplasms: Adenoma, Carcinoma or Adenoma with Neuro- 
endocrine Differentiation: A Case Series. Cases Journal, 2, 6508.

http://dx.doi.org/10.1186/1757-1626-0002-0000006508

[5] Batsakis, J.G. (1989) Adenomatous Tumours of the Middle Ear. Annals of Otology, Rhinology, and Laryngology, 98, 749-752. 